This item was submitted to Loughborough's Research Repository by the author.

Items in Figshare are protected by copyright, with all rights reserved, unless otherwise indicated.

\title{
A novel adaptive algorithm for the blind separation of periodic sources
}

PLEASE CITE THE PUBLISHED VERSION

PUBLISHER

(C) IEEE

VERSION

VoR (Version of Record)

LICENCE

CC BY-NC-ND 4.0

\section{REPOSITORY RECORD}

Jafari, Maria G., and Jonathon Chambers. 2019. "A Novel Adaptive Algorithm for the Blind Separation of Periodic Sources”. figshare. https://hdl.handle.net/2134/5668. 
This item was submitted to Loughborough's Institutional Repository (https://dspace.lboro.ac.uk/) by the author and is made available under the following Creative Commons Licence conditions.

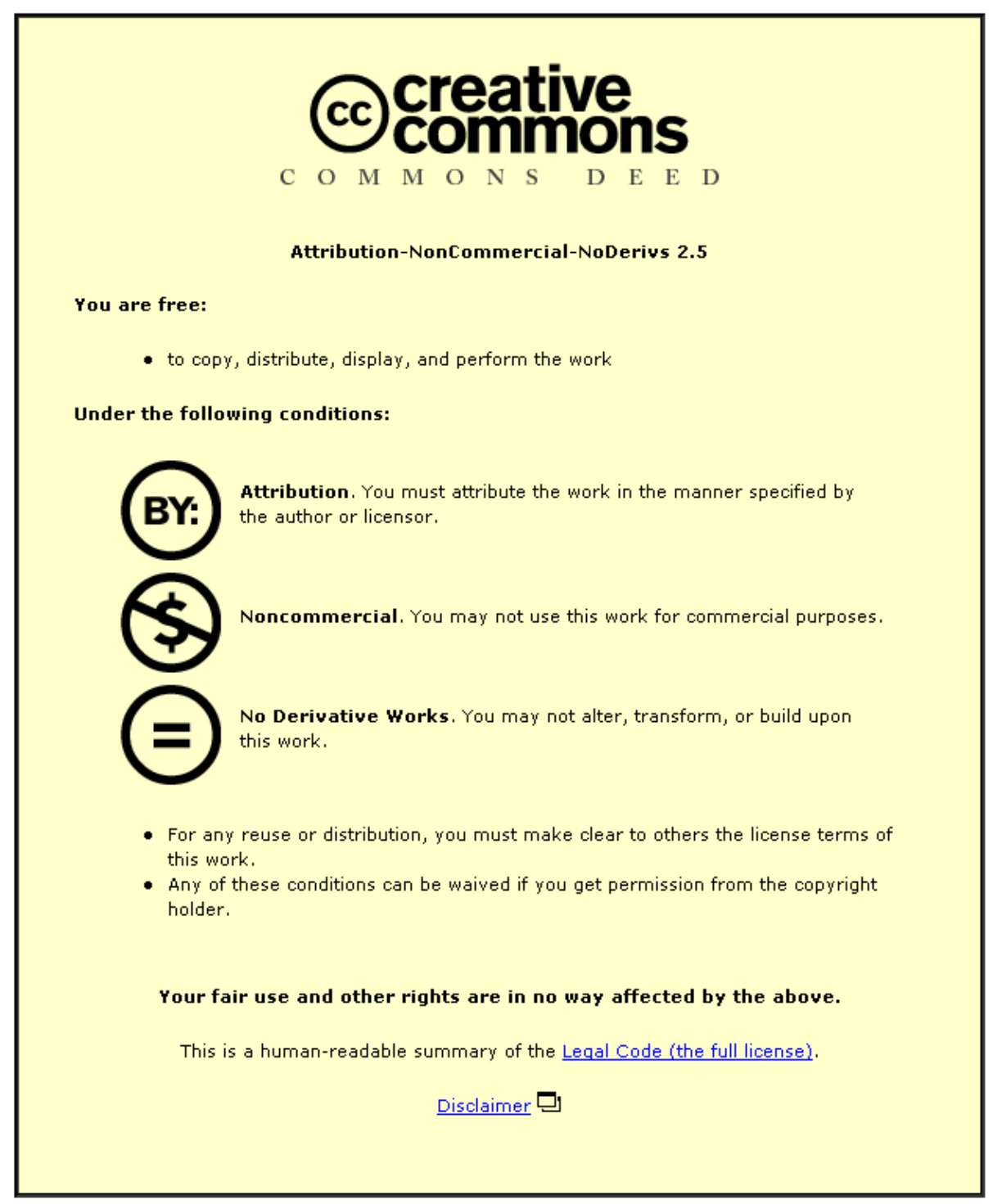

For the full text of this licence, please go to: http://creativecommons.org/licenses/by-nc-nd/2.5/ 


\section{A novel adaptive algorithm for the blind separation of periodic sources}

\author{
M. G. Jafari \\ Centre for Digital Signal Processing Research \\ King's College London \\ Strand, London WC2R 2LS, U.K. \\ E-mail: maria.jafari@kcl.ac.uk
}

\author{
J. A. Chambers \\ Cardiff School of Engineering \\ Cardiff University \\ Cardiff CF24 OYF, U.K. \\ E-mail: chambersj@cf.ac.uk
}

\begin{abstract}
An adaptive algorithm for the blind separation of periodic sources is proposed in this paper. The method uses only the second order statistics of the data, and exploits the periodic nature of the source signals. Simulation results show that the proposed approach has the ability to restore statistical independence, and its performance is comparable to that of a well established, higher order, blind source separation method.
\end{abstract}

\section{INTRODUCTION}

Blind source separation (BSS) addresses the problem that arises when a set of unobservable source signals must be recovered from a set of measurements, generated when the original signals are mixed by an unknown medium. The blind source separation problem is typically solved by exploiting the second or higher order statistics of the data, with higher order methods generally representing the preferred approach, since they require few assumptions other than the statistical independence of the sources. For this reason they have resulted in a large number of algorithms (e.g. [1], [2], [3], [4], [5]). Conversely, second order methods require shorter data records, and do not limit the number of Gaussian sources that can be separated to one, but their derivation is usually based on additional assumptions made on the nature of the original signals, such as statistical non-stationarity of the sources [6], [7], [8], presence of time correlations in stationary signals [9], [10], [11], or cyclostationarity [12], [13], [14].

A novel adaptive algorithm, for the blind separation of periodic source signals, based only on second order statistical information is proposed in this paper. Source separation is performed by diagonalising the output autocorrelation matrix at a lag $\tau$ corresponding to the fundamental period of periodicity of one of the source signals. Although the method requires that the delay $\tau$ is equal to the fundamental period of one of the sources, it will successfully separate the sources even when the selected lag does not correspond to the exact period.

Thus, we begin by stating the blind source separation method in Section I-A. The identification principle on which the proposed algorithm is based is described in Section II, while the sequential algorithm is introduced in Section
III. The performance of the proposed method is shown by simulation in Section IV, and conclusions are drawn in Section V.

\section{A. Problem statement}

The aim of BSS methods is to recover the source signals $\mathbf{s}(k) \in \mathbb{C}^{m}$, from the observed mixtures $\mathbf{x}(k) \in \mathbb{C}^{n}$, which are generated by the mixing model

$$
\mathbf{x}(k)=\mathbf{A s}(k)
$$

where $\mathbf{A} \in \mathbb{C}^{n \times m}$ is an unknown, full column rank, mixing matrix, and $k$ is the discrete time index. The original sources, assumed to be zero mean, unit variance, and mutually statistically uncorrelated, are then estimated according to the separating model

$$
\mathbf{y}(k)=\mathbf{W}(k) \mathbf{x}(k)
$$

where $\mathbf{y}(k) \in \mathbb{C}^{m}$ represents the recovered sources, and $\mathbf{W}(k) \in \mathbb{C}^{m \times n}$ is the separating matrix.

In this paper, it is also assumed that the sources are temporally periodic, such that the $i$-th source, with fundamental period $T_{i}$, can be written as

$$
s_{i}(k)=s_{i}\left(k+n T_{i}\right)
$$

where $n \in \mathbb{Z}$, and $\mathbb{Z}$ denotes the field of integers. Periodicity of the source implies that its autocorrelation function, defined as a function of lag $\tau, \rho_{s_{i}}(\tau)=\left\langle s_{i}(k) s_{i}^{*}(k+\tau)\right\rangle$, is also periodic with period $T_{i}$, that is

$$
\rho_{s_{i}}(\tau)=\rho_{s_{i}}\left(\tau+n T_{i}\right)=\left\langle s_{i}(k) s_{i}^{*}\left(k+\tau+n T_{i}\right)\right\rangle
$$

where $(\cdot)^{*}$ and $\langle\cdot\rangle$ represent, respectively, complex conjugation and statistical expectation. Thus, the autocorrelation matrix of the source signals, at a lag $\tau=T_{i}$ becomes

$$
\mathbf{R}_{s}\left(T_{i}\right)=\left\langle\mathrm{s}(k) \mathrm{s}^{H}\left(k+T_{i}\right)\right\rangle
$$

where $(\cdot)^{H}$ denotes the Hermitian transpose operator. From (4), the elements of the matrix in (5) are given by

$$
\left\langle s_{i}(k) s_{j}^{*}\left(k+T_{j}\right)\right\rangle= \begin{cases}0, & \forall j \neq i \\ 1, & \forall j=i, T_{j}=n T_{i}(6) \\ \rho_{s_{i}}\left(T_{j}\right), & \forall j=i, T_{j} \neq n T_{i}\end{cases}
$$


where $n \in \mathbb{Z}$. Thus, the source autocorrelation matrix at lag $T_{i}$ is given by

$$
\mathbf{R}_{s}\left(T_{i}\right)=\Lambda_{s}\left(T_{i}\right)
$$

where $\Lambda_{s}\left(T_{i}\right)$ is the eigenvalue matrix of $\mathbf{R}_{s}\left(T_{i}\right)$, whose elements are defined in (6). Note that the source autocorrelation matrix is not guaranteed to be positive definite. In this paper, we do assume, for algorithm development, that the eigenvalues of the source autocorrelation matrix at the delay $\tau=T_{i}$ are distinct and non-zero.

\section{IDENTIFICATION PRINCIPLE}

The output autocorrelation matrix evaluated at a lag $\tau=T_{i}$ is given by

$$
\begin{aligned}
\mathbf{R}_{y}\left(k, T_{i}\right) & =\left\langle\mathbf{y}(k) \mathbf{y}^{H}\left(k+T_{i}\right)\right\rangle \\
& =\left\langle\mathbf{W}(k) \mathbf{A} \mathbf{s}(k) \mathbf{s}^{H}\left(k+T_{i}\right) \mathbf{A}^{H} \mathbf{W}^{H}\left(k+T_{i}\right)\right\rangle \\
& =\mathbf{P}(k)\left\langle\mathbf{s}(k) \mathbf{s}^{H}\left(k+T_{i}\right)\right\rangle \mathbf{P}^{H}\left(k+T_{i}\right) \\
& =\mathbf{P}(k) \boldsymbol{\Lambda}_{s}\left(T_{i}\right) \mathbf{P}^{H}\left(k+T_{i}\right)
\end{aligned}
$$

where $\mathbf{P}(k)=\mathbf{W}(k) \mathbf{A}$ is the global mixing-separating matrix. The above expression allows the derivation of a periodic decorrelation algorithm, which has also the ability of effectively performing blind source separation on the basis of only second order statistical information. To see this, let $\mathbf{R}_{y}^{o}\left(T_{i}\right)$ denote the output autocorrelation matrix at convergence, that is

$$
\lim _{k \rightarrow \infty} \mathbf{R}_{y}\left(k, T_{i}\right)=\mathbf{R}_{y}^{o}\left(T_{i}\right)
$$

By definition, the whitening operation implies that

$$
\mathbf{R}_{y}^{o}\left(T_{i}\right)=\mathbf{P}(k) \boldsymbol{\Lambda}_{s}\left(T_{i}\right) \mathbf{P}^{H}(k)=\hat{\mathbf{D}}\left(T_{i}\right)
$$

where $\hat{\mathbf{D}}\left(T_{i}\right)$ is a diagonal matrix and, since the system (10) approaches steady-state, $\mathbf{P}(k) \approx \mathbf{P}\left(k+T_{i}\right)$. Equation (10) implies that $\mathbf{P}(k)$ is a unitary matrix which can diagonalise the output autocorrelation matrix provided that its eigenvalues are distinct and non-zero. Moreover, the columns of $\mathbf{P}(k)$ are the eigenvectors of $\mathbf{R}_{y}^{o}\left(T_{i}\right)$.

The matrix $\mathbf{R}_{y}\left(k, T_{i}\right)$ converges to $\hat{\mathbf{D}}\left(T_{i}\right)$ rather than $\boldsymbol{\Lambda}_{s}\left(T_{i}\right)$ because of the indeterminacies of scaling and permutation, which are due to the non-uniqueness of the eigenvectors contained in $\mathbf{P}(k)$. Thus, $\hat{\mathbf{D}}\left(T_{i}\right)$ estimates a matrix whose diagonal elements are the eigenvalues of $\mathbf{R}_{y}\left(k, T_{i}\right)$ within a scaling and permutation ambiguity. Also, it should be noted that $\mathbf{P}(k)$ is the eigenvector matrix of $\mathbf{R}_{y}\left(k, T_{i}\right)$ and not $\mathbf{R}_{s}\left(T_{i}\right)$.

\section{PERIODIC ALGORITHM}

A sequential algorithm for the decorrelation of periodic signals can be derived by minimising the following information theoretic criterion [15]

$$
\begin{aligned}
J(\mathbf{W}(k))= & -\frac{1}{2} \log \left(\operatorname{det}\left(\mathbf{W}(k) \mathbf{W}^{H}(k)\right)\right) \\
& +\frac{1}{2} \sum_{i=1}^{m}\left\langle\left|y_{i}(k) y_{i}^{*}(k+\tau)\right|\right\rangle
\end{aligned}
$$

where $|\cdot|$ denotes the absolute value. Differentiation of the first term on the right hand side of (11) with respect to the separating matrix $\mathbf{W}(k)$ leads to

$$
\frac{\partial \log \left(\operatorname{det}\left(\mathbf{W}(k) \mathbf{W}^{H}(k)\right)\right)}{\partial \mathbf{W}(k)}=2\left(\mathbf{W}(k) \mathbf{W}^{H}(k)\right)^{-1} \mathbf{W}(k)
$$

Following the approach in [15], it can be shown that differentiation of the second term on the right hand side of (11) leads to

$$
\begin{gathered}
\frac{\partial\left\langle y_{i}(k) y_{i}^{*}(k+\tau)\right\rangle}{\partial w_{i j}(k)} \approx\left\langle y_{i}^{*}(k+\tau) x_{j}(k)\right\rangle+\left\langle y_{i}(k) x_{j}^{*}(k+\tau)\right\rangle \\
\forall i, j=1, \ldots, m
\end{gathered}
$$

where it has been assumed that

$$
w_{i j}(k) \approx w_{i j}(k+\tau)
$$

In matrix form, (13) becomes

$$
\begin{aligned}
\frac{\partial \sum_{i=1}^{m}\left\langle\left|y_{i}(k) y_{i}^{*}(k+\tau)\right|\right\rangle}{\partial \mathbf{W}(k)} \approx & \left\langle\mathbf{y}(k+\tau) \mathbf{x}^{H}(k)\right. \\
& \left.+\mathbf{y}(k) \mathbf{x}^{H}(k+\tau)\right\rangle
\end{aligned}
$$

Employing the natural gradient descent method ${ }^{1}$, the gradient of (11) is obtained from (12) and (15) as

$$
\begin{aligned}
\nabla J(\mathbf{W}(k))= & \frac{\partial J(\mathbf{W}(k))}{\partial \mathbf{W}(k)} \mathbf{W}^{H}(k) \mathbf{W}(k) \\
=- & {\left[\mathbf{I}-\frac{1}{2}\left\langle\mathbf{y}(k+\tau) \mathbf{y}^{H}(k)\right.\right.} \\
& \left.\left.+\mathbf{y}(k) \mathbf{y}^{H}(k+\tau)\right\rangle\right] \mathbf{W}(k)
\end{aligned}
$$

where the assumption (14) has again been used, and for convenience, the approximation in (14) is not explicitly shown. In terms of the output periodic autocorrelation function (8), (17) leads to the following learning rule

$\mathbf{W}(k+1)=\mathbf{W}(k)+\eta\left[\mathbf{I}-\frac{\mathbf{1}}{2}\left(\mathbf{R}_{y}^{H}\left(k, T_{i}\right)+\mathbf{R}_{y}\left(k, T_{i}\right)\right)\right] \mathbf{W}(k)$

where $\eta$ is a step-size parameter.

However, the algorithm (18) will not separate the sources when $\mathbf{R}_{y}\left(k, T_{i}\right)$ is not positive definite, since the direction of descent may vary during the adaptive procedure, causing some of the elements of the estimated separating matrix to oscillate between positive and negative values. Thus, following the approach in [15], we replace $\mathbf{R}_{y}\left(k, T_{i}\right)$ in (18) with $\mathbf{R}_{y}\left(k, T_{i}\right) \mathbf{S}\left(\mathbf{y}\left(k, T_{i}\right)\right)$, where

$$
\left[\mathbf{S}\left(\mathbf{y}\left(k, T_{i}\right)\right)\right]_{i, j}= \begin{cases}\frac{\left\langle y_{i}(k) y_{i}\left(k+T_{i}\right)\right\rangle}{\left|\left\langle y_{i}(k) y_{i}\left(k+T_{i}\right)\right\rangle\right|}, & \text { if } i=j \\ 0, & \text { otherwise }\end{cases}
$$

\footnotetext{
${ }^{1}$ The Atick-Redlich formula can also be used here. In this case, the same gradient is obtained using [15]

$$
\nabla J(\mathbf{W}(k))=\mathbf{W}(k)\left[\frac{\partial J(\mathbf{W}(k))}{\partial \mathbf{W}(k)}\right]^{T} \mathbf{W}(k)
$$


Hence, (18) becomes

$$
\begin{aligned}
\mathbf{W}(k+1)= & \mathbf{W}(k)+\eta\left\{\mathbf{I}-\frac{1}{2}\left[\mathbf{R}_{y}^{H}\left(k, T_{i}\right) \mathbf{S}\left(\mathbf{y}\left(k, T_{i}\right)\right)\right.\right. \\
& \left.\left.+\mathbf{R}_{y}\left(k, T_{i}\right) \mathbf{S}\left(\mathbf{y}\left(k, T_{i}\right)\right)\right]\right\} \mathbf{W}(k)
\end{aligned}
$$

where it can be shown that the matrix $\mathbf{R}_{y}\left(k, T_{i}\right) \mathbf{S}\left(\mathbf{y}\left(k, T_{i}\right)\right)$ is guaranteed to be positive definite.

It should be noted that the matrix $\mathbf{R}_{y}^{H}\left(k, T_{i}\right)+\mathbf{R}_{y}\left(k, T_{i}\right)$ is Hermitian, which implies that it does not offer enough degrees of freedom for source separation [16]. Conversely, $\mathbf{R}_{y}^{H}\left(k, T_{i}\right) \mathbf{S}\left(\mathbf{y}\left(k, T_{i}\right)\right)+\mathbf{R}_{y}\left(k, T_{i}\right) \mathbf{S}\left(\mathbf{y}\left(k, T_{i}\right)\right)$ is not Hermitian, because both $\mathbf{R}_{y}\left(k, T_{i}\right)$ and $\mathbf{R}_{y}^{H}\left(k, T_{i}\right)$ are multiplied by $\mathbf{S}\left(\mathbf{y}\left(k, T_{i}\right)\right)$, thus ensuring that a sufficient number of degrees of freedom is preserved for the solution of the BSS problem.

For the practical implementation of $(20)$, the matrices $\mathbf{R}_{y}\left(k, T_{i}\right)$ and $\mathbf{S}\left(\mathbf{y}\left(k, T_{i}\right)\right)$ are replaced by their instantaneous estimate, given by

$$
\hat{\mathbf{R}}_{y}\left(k, T_{i}\right)=\mathbf{y}(k) \mathbf{y}^{H}\left(k+T_{i}\right)
$$

and where

$$
\left[\hat{\mathbf{S}}\left(\mathbf{y}\left(k, T_{i}\right)\right)\right]_{i, i}= \begin{cases}\frac{y_{i}(k) y_{i}\left(k+T_{i}\right)}{\left|y_{i}(k) y_{i}\left(k+T_{i}\right)\right|}, & \text { if } i=j \\ 0, & \text { otherwise }\end{cases}
$$

so that the algorithm (20) becomes

$$
\begin{aligned}
\mathbf{W}(k+1)= & \mathbf{W}(k)+\eta\left\{\mathbf{I}-\frac{1}{2}\left[\hat{\mathbf{R}}_{y}^{H}\left(k, T_{i}\right) \hat{\mathbf{S}}\left(\mathbf{y}\left(k, T_{i}\right)\right)\right.\right. \\
& \left.\left.+\hat{\mathbf{R}}_{y}\left(k, T_{i}\right) \mathbf{S}\left(\mathbf{y}\left(\mathbf{k}, \mathbf{T}_{\mathbf{i}}\right)\right)\right]\right\} \mathbf{W}(k)
\end{aligned}
$$

The algorithm proposed here has the ability to separate source signals for any arbitrary value of the lag $\tau$. Thus, although the method has been derived for the case of temporally periodic sources, provided that the source autocorrelation function is non-zero, and that the sources have different spectral characteristics, the assumption that the lag equals the fundamental period of periodicity can be relaxed.

\section{SiMULATION RESULTS}

The performance of the proposed method was compared to that of the well established EASI algorithm, given by [5]

$$
\begin{aligned}
\mathbf{W}(k+1)= & \mathbf{W}(k)+\mu\left\{\mathbf{I}-\mathbf{y}(k) \mathbf{y}^{T}(k)+\mathbf{y}(k) \mathbf{f}^{T}(\mathbf{y}(k))\right. \\
& \left.-\mathbf{f}(\mathbf{y}(k)) \mathbf{y}^{T}(k)\right\} \mathbf{W}(k)
\end{aligned}
$$

where $\mu$ is a positive step-size parameter, and $\mathbf{f}(\mathbf{y}(k))$ is an odd non-linear function of the output $\mathbf{y}(k)$.

Firstly, a sinusoidal source $s_{1}(k)$ with fundamental period $T_{1}=100$ samples and a signal $s_{2}(k)$ uniformly distributed in $[-1,1]$, both shown in Fig. 1, were mixed by a time-invariant $2 \times 2$ mixing matrix, so that the mixtures $x_{1}(k)$ and $x_{2}(k)$ were generated. Separation was performed with (23), when

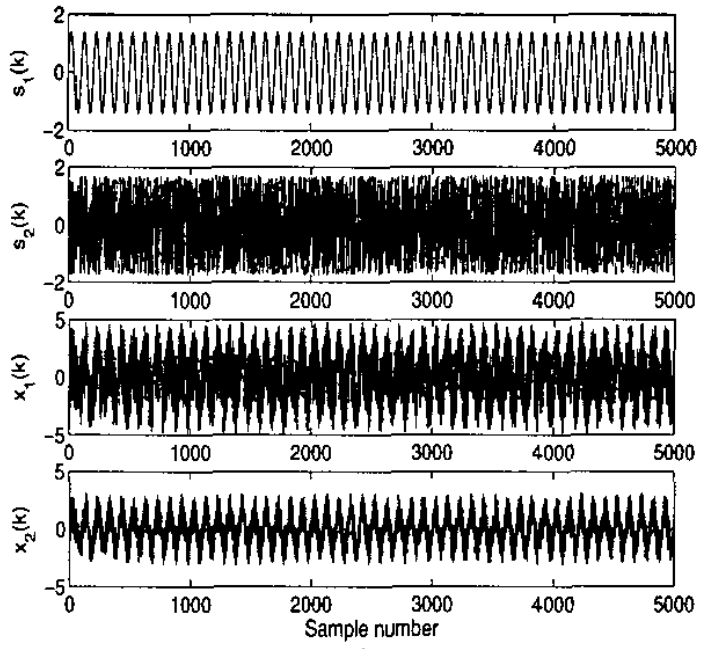

Fig. 1. Original source signals $\left(s_{1}(k)\right.$ and $\left.s_{2}(k)\right)$, and mixed signals $\left(x_{1}(k)\right.$ and $\left.x_{2}(k)\right)$.

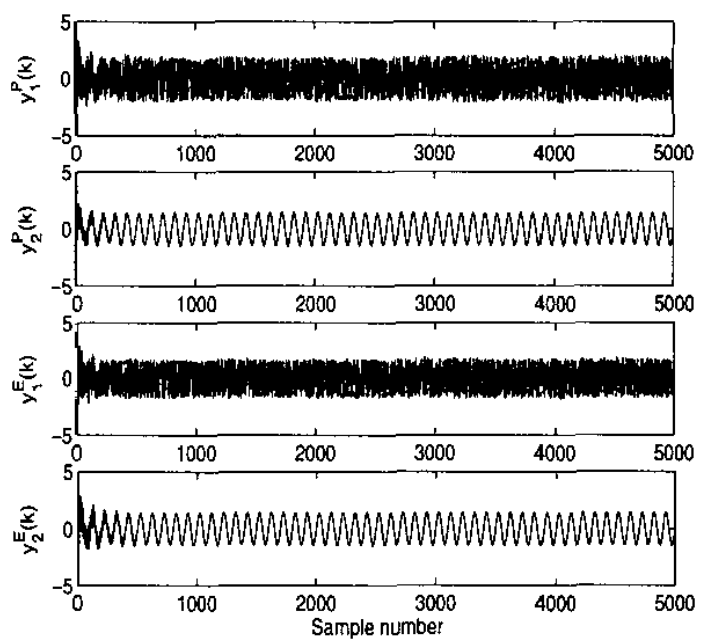

Fig. 2. Signals recovered with the EASl algorithm $\left(y_{1}^{E}(k)\right.$ and $\left.y_{2}^{E}(k)\right)$, and with the algorithm $(23)\left(y_{1}^{P}(k)\right.$ and $\left.y_{2}^{P}(k)\right)$.

$\eta=0.003, T_{i}=100$, and with the EASI algorithm (24), when $\mu=0.003$, and $f_{i}\left(y_{i}(k)\right)=y_{i}^{3}(k)$. In Fig. $2 y_{1}^{E}(k)$ and $y_{2}^{E}(k)$ represent the signals recovered with the EASI algorithm, and $y_{1}^{P}(k)$ and $y_{2}^{P}(k)$ are the outputs of the proposed algorithm. The results illustrate that the proposed method recovers the original components, and preserves waveform similarity in a similar fashion as the EASI algorithm.

The two upper plots in Fig. 3 depict the support of the joint probability density function (pdf) of the source signals (upper-left plot), and the mixtures (upper-right). The support of the joint pdf of the sources separated with the proposed periodic BSS algorithm and with EASI, following initial 

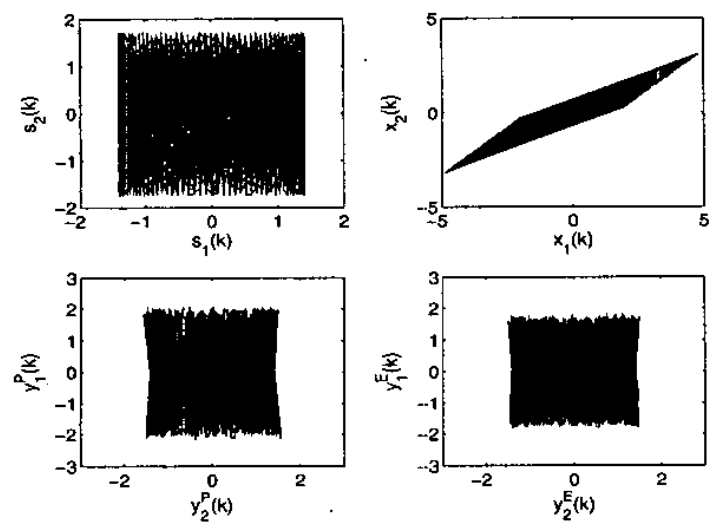

Fig. 3. Scatter plots of the source signals (upper-left plot), the mixtures (upper-right plot), and the outputs of the proposed algorithm (23) $\left(y_{1}^{P}(k)\right.$ and $y_{2}^{P}(k)$ ) (lower-left plot), and EASI $\left(y_{1}^{E}(k)\right.$ and $y_{2}^{E}(k)$ ) (lower-right plot).

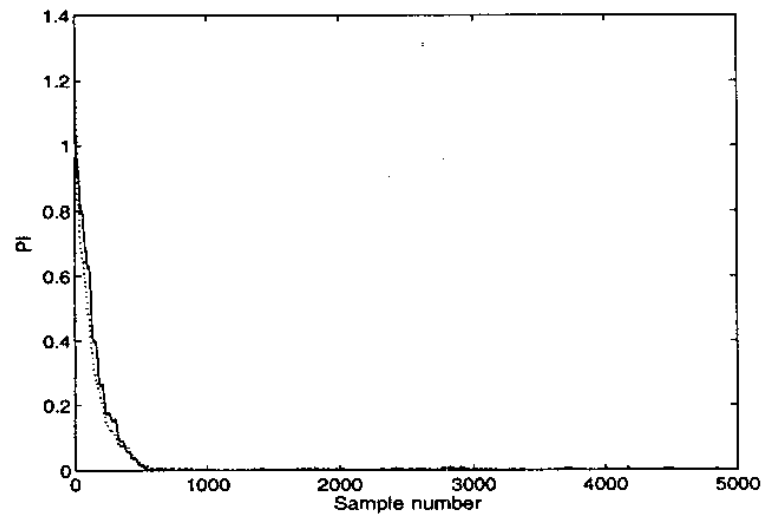

Fig. 4. Performance indices obtained with the algorithm (23) and with the EASl algorithm (24), for a single realisation.

convergence of the algorithm (i.e., for $500 \leq k \leq 5000$ ), are depicted,respectively, in the lower-left and lower-right plots.

The shape of the joint distribution of the source signals clearly indicates that they are statistically independent, since the knowledge of the value of one does not convey any information about the value of the other. The signals generated by the mixing procedure, $x_{1}$ and $x_{2}$, are no longer independent, and the joint pdf now has the form of a parallelogram. The results depicted in the lower plots of Fig. 1 , show that the periodic decorrelation algorithm proposed here has the necessary degrees of freedom to allow it to rotate the joint pdf of the recovered sources, as well as decorrelate the signals, leading to the restoration of statistical independence.

The performance of the periodic BSS algorithm (23) and EASI (24) was compared in terms of the performance index (PI), as defined in [17], where generally a low PI indicates better performance. The performance indices for the two methods are compared in Fig. 4, which illustrates that the proposed algorithm is capable of separating the source signals, with a performance comparable to the behaviour of EASI.
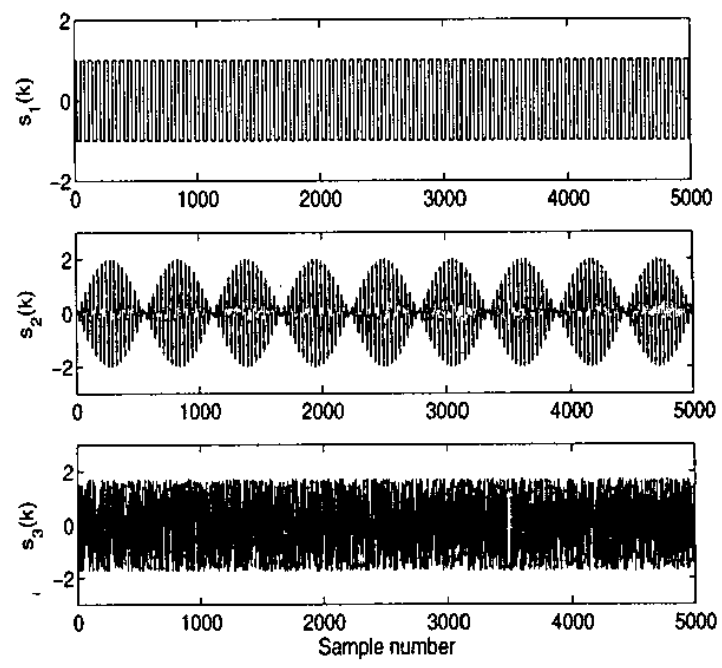

Fig. 5. Original source signals $s_{1}(k), s_{2}(k)$, and $s_{3}(k)$.

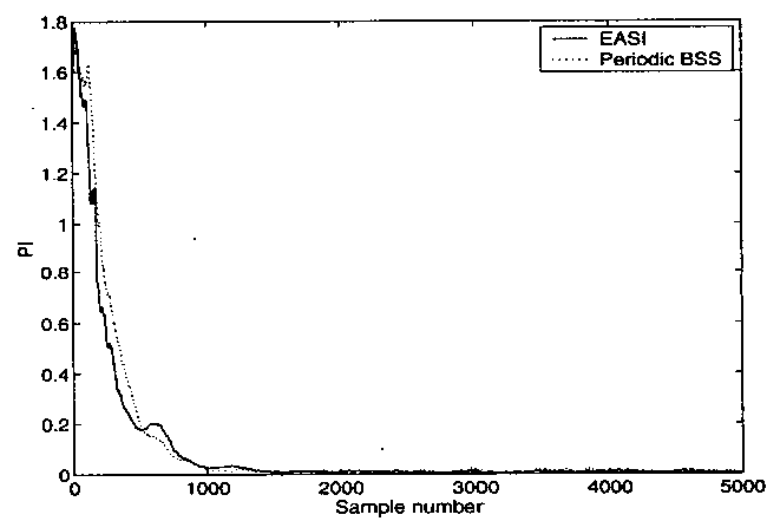

Fig. 6. Performance index obtained with the proposed periodic BSS algorithm and EASI, averaged over 100 independent trials.

Next, the average performance of the proposed algorithm is investigated. Fig. 5 shows a signal uniformly distributed in $[-1,1]$, a sign and an amplitude-modulated signal, given by

$$
\begin{aligned}
& s_{1}(k)=\operatorname{sgn}\left(\cos \left(2 \pi f_{1} t_{s} k\right)\right) \\
& s_{2}(k)=\sin \left(2 \pi f_{2} t_{s} k\right) \sin \left(2 \pi f_{3} t_{s} k\right)
\end{aligned}
$$

where $t_{s}=9 \times 10^{-4}, f_{1}=155 \mathrm{~Hz}, f_{2}=9 \mathrm{~Hz}$, and $f_{3}=$ $300 \mathrm{~Hz}$. The sources were mixed by a stationary $3 \times 3$ mixing matrix, and noise was added such that the signal to noise ratio was $5 \mathrm{~dB}$. Separation was performed with (23), when $\eta=0.003, T_{i}=\left(f_{2}+f_{3}\right)^{-1}$ where $\left(f_{2}+f_{3}\right)$ is the upper 
side band of source $s_{2}(k)$, and with the EASI algorithm (24), when $\mu=0.003$. The PI resulting from the application of the two methods, and averaged over 100 independent trials, is shown in Fig. 6. It illustrates that the behaviour of the proposed method is similar to that of EASI, both during and following initial convergence.

\section{CONCLUSIONS}

In this paper a novel sequential algorithm for the blind separation of periodic source signals is introduced, which exploits the temporal periodic nature of the source signals, and thus performs separation based only on second order statistics. Computer simulations have shown that the method allows the recovery of the original sources, and restoration of statistical independence. Moreover, its behaviour is comparable to that of EASI, during and following initial convergence.

\section{REFERENCES}

[1] S. Amari, A. Cichocki, and H. H. Yang, "A new leaming algorithm for blind signal separation," in Advances in Neural Information Processing Systems, vol. 8, 1996, pp. 752-763.

[2] C. Jutten and J. Herault, "Blind separation of sources, PartI: An adaptive algorithm based on a neuromimetic architecture," Signal Processing, vol. 24, pp. 1-10, 1991.

[3] P. Comon, "Independent component analysis, a new concept?" Signal Processing, vol. 36, pp. 287-314, 1994.

[4] A. J. Bell and T. J. Sejnowski, "An information-maximisation approach to blind separation and blind deconvolution," Neural Computation, vol. 7, pp. 1129-1 159, 1995.

[5] J. F. Cardoso and B. Laheld, "Equivariant adaptive source separation," IEEE Truns. on Signal Processing, vol. 44, pp. 3017-3030, 1996.

[6] K. Matsuoka, M. Ohya, and M. Kawamoto, "A neural net for blind separation of nonstationary signals," Neural Networks, vol. 8, pp. 411 419, 1995.

[7] S. Choi and O. Y. Lee, "Nonstationary source separation," in Proc. of the IEEE Region IO Conference, vol. 1, 1999, pp. 670-673.

[8] D. L. Jones, "A new method for blind source separation of nonstationary signals," in Proc. of the IEEE Int. Conf. on Acoustic, Speech, and Signal Processing, vol. 5, 1999, pp. 2893-2896.

[9] A. Belouchrani, K. Abed-Meraim, J. Cardoso, and E. Moulines, "A blind source separation technique using second-order statistics," IEEE Trans. on Signal Processing, vol. 45, pp. 434-444, 1997.

[10] S. Choi and A. Cichock, "Blind separation of nonstationary sources in noisy mixtures," Electronics Letters, vol. 36, pp. 848-849, 2000.

[11] A. Belouchrani and A. Cichocki, "A robust procedure in blind source separation context," Electronics Letters, vol. 36, pp. 2050-2051, 2000

[12] A. Ferreol and P. Chevalier, "On the behaviour of current second and higher order blind source separation methods for cyclostationary sources," IEEE Trans. on Signal Processing, vol. 48, pp. 1712-1725, 2000.

[13] K. Abed-Meraim, Y. Xiang, J. H. Manton, and Y. Hua, "Blind source separation using second-order cyclostationary statistics," IEEE Trans. on Signal Processing, vol. 49, pp. 694-701, 2001.

[14] M. G. Jafari and J. A. Chambers, "A new natural gradient algorithm for cyclostationary sources," To appear: IEE Proceedings Vision, Image and Signal Proces:ing, 2002.

[15] A. Cichocki and S. Amari, Adaptive Blind Signal and Image Processing: Learning Algorithns and Applications. John Wiley, 2002.

[16] J. F. Cardoso, "Blind signal separation: statistical principles," Proceedings of the IEEE, vol. 86, pp. 2009-2025, 1998.

[17] A. Cichocki, B. Drsier, A. Back, and S. Amari, "On-line adaptive algorithms in non-stationary environments using a modified conjugate gradient approach," in Proc. of the IEEE Workshop on Neural Networks for Signal Processing. 1997, pp. 316-325. 